\title{
Grafen oksitin modifiye Hummers yöntemi ile sentezi ve film olarak Al/GO/n- InP diyot performansına etkileri
}

\author{
Synthesis of graphene oxide by modified Hummers method and its effects on Al/GO/n-InP \\ diode performance as interlayer film
}

Fulya Esra CIMILLİ ÇATIR*1

${ }^{1}$ Erzincan Binali Yıldırım Üniversitesi, Meslek Yüksek Okulu, Elektronik ve Otomasyon Bölümü,24000, Erzincan

• Geliş tarihi / Received: 15.07.2020 • Düzeltilerek geliş tarihi / Received in revised form: $30.11 .2020 \quad$ • Kabul tarihi / Accepted: 31.12 .2020

\section{$\ddot{O} z$}

Grafen oksit (GO) modifiye Hummers yöntemi ile sentezlendi. Metal-yariletken arayüzeyine spray pyrolysis yöntemiyle büyütülen grafen oksit filmin $\mathrm{Al} / \mathrm{GO} / \mathrm{n}$-InP Schottky diyot karakteristikleri üzerine etkisi araştırıldı. GO filmlerin yapısal özellikleri X-Işını kırınımı ölçümleri (XRD) ve taramalı elektron mikroskobu (SEM) ölçümleri ile belirlendi. GO filmin absorbans ve transmittans spektrumları alınarak optik özellikleri araştırıldı. Al/GO/n-InP diyotunun oda sıcaklığındaki I$V$ karakteristiklerinden bu yapının doğrultucu özellik gösterdiği görüldü. Ayrıca, karanlık ve $100 \mathrm{~mW} / \mathrm{cm}^{2}$ 1şık şiddeti altında yapılan $I-V$ ölçümleri doğrultusunda diyotun oldukça iyi fotovoltaik özelliklere sahip olduğu belirlendi. Al/GO/nInP Schottky diyotunun idealite faktörü $(n)$, engel yüksekliği $\left(\Phi_{b 0}\right)$, seri direnç $\left(R_{s}\right)$ ve şönt direnci $R_{s h}$ gibi karakteristik parametreleri hesaplanarak arayüzey tabakasız referans $\mathrm{Al} / \mathrm{n}$-InP diyotu ile karşılaştırıldı. Al/GO/n-InP Schottky diyot yapısının çeşitli elektronik ve optoelektronik devre uygulamaları için uygun bir malzeme olduğu görüldü.

Anahtar kelimeler: Absorbans, Grafen oksit, $I$ - $V$ ölçümleri, n-InP, SEM, Transmittans, XRD

\begin{abstract}
Graphene oxide (GO) was synthesized by the modified Hummers method. The graphene oxide film deposited by using spray pyrolysis on metal-semiconductor interface of Al/GO/n-InP Schottky diode. The effect of GO film on Al/GO/n-InP Schottky diode characteristics was investigated. The structural properties of GO films were determined by X-Ray diffraction measurements (XRD) and scanning electron microscopy (SEM) measurements. The absorbance and transmittance spectra of the GO film were measured and their optical properties were investigated. From the I-V characteristics of the Al/GO/n-InP diode at room temperature, this structure was shown to have a rectifying property. In addition, it was determined that the diode had very good photovoltaic properties from the I-V measurements under dark and $100 \mathrm{~mW} / \mathrm{cm} 2$ light intensity. The characteristic parameters of the Al/GO/n-InP Schottky diode such as the ideal factor $(n)$, barrier height ( $\Phi b 0)$, series resistance (Rs) and shunt resistance (Rsh) were calculated and compared with the reference Al/n-InP diode. The Al/GO/n-InP Schottky diode structure was found to be a suitable material for various electronic and optoelectronic circuit applications.
\end{abstract}

Keywords: Absorbance, Graphene oxide, I-V measurements, $n$-InP, Transmittance, XRD, SEM

\footnotetext{
*a Fulya Esra CIMILLİ ÇATIR; fcatir@erzincan.edu.tr, Tel: (0446) 22666 00, orcid.org/0000-0002-0757-3130
} 


\section{Giriş}

Grafen oksit (GO), bilim ve teknolojinin birçok dalında araştırmacıların ilgisini çeken çok yönlü bir malzemedir (Perrozzi vd., 2014). GO'in, üç boyutlu yapıs1, son derece yüksek, yüzey alan1, ayarlanabilir elektriksel iletkenlik, iyi kimyasal/elektrokimyasal kararlılık, yüksek esneklik, orta derecede iletkenlik, yüksek kimyasal kararlılık ve mükemmel mekanik, optik, termal, elektrokimyasal özellikler göstermektedir. $\mathrm{Bu}$ nedenle GO, gaz sensörleri veya depolama, yüksek performansli fiberler, kompozit membranlar, elektrokimyasal uygulamalar, enerji cihazları, alan etkili transistörler ve benzeri gibi çok çeşitli uygulamalarda arzu edilen bir malzeme olarak kabul edilmektedir (Chen vd., 2014; Li vd., 2015).

GO homojen bir malzeme değildir ve yapısı iyi tanımlanmamıştır. $\mathrm{Bu}$ nedenle, iletkenlik büyük ölçüde sentez yöntemine bağlı olacaktır. Grafenle karşılaştırıldığında yapısında değişik oranlarda hidrojen, oksijen ve karbon bulunduran grafen oksit, literatürde yaygın olarak Brodie, Staundenmaier, Offeman ve Hummers yöntemleri ile elde edilmiştir. Grafen oksit (GO) yapısı, grafenin yoğun bir şekilde kuvvetli oksitleyicilerle yükseltgenmiş halidir. Grafen oksit, oksitleyici maddeler grafit ile reaksiyona girdiğinde, grafit katmanları arasındaki düzlemler arası boşluk arttığında oluşan bu oksitlenmenin bir yan ürünüdür. Tamamen oksitlenmiş bileșik daha sonra su veya uygun bir baz çözeltisi içinde dağıtılabilir ve daha sonra grafen oksit üretilir. Grafen oksit üretiminde en eski ve en yaygın kullanılan yöntemlerden biri ise Hummers metodudur. Hummers metodu ile sentezlenen GO, oda sıcaklığında yalıtkandır ancak, iletkenliği yapısında bulunan oksijenin azaltılmasi ile artmaktadır (Park ve Ruoff, 2009).

GO, yapısında karbon, oksijen ve hidrojen atomlarının yanı sıra hidroksil, epoksi ve karboksil gibi fonksiyonel gruplar bulundurmaktadır (Baltakesmez vd., 2019). Öte yandan, bu oksijen fonksiyonel grupları elektriksel olarak iletken olan grafeni bir yalitkana dönüştüren kusurlar olarak düşünülebilir. Bununla birlikte, oksijen işlevleri GO'e grafenin sahip olmadığ 1 birçok benzersiz özellik sağlar. $\mathrm{Bu}$ özelliklerden biri hidrofilik olması, yani suda ve bazı düşük moleküler ağırlıklı alkollerde çözünebilir olması ve kararlı kolloid çözeltileri oluşturmasının yanında, ona benzersiz optik ve elektronik özellikler sağlayan ayarlanabilir bir bant aralığına sahip olmasıdır (Dimiev ve Eigler, 2016; Dimiev ve Tour, 2014; Sattler, 2016; Zhao vd., 2015).
$\mathrm{C} / \mathrm{O}$ oranı 2/1 olan grafen oksit iletken değildir. Genellikle mikro $\mathrm{S} / \mathrm{m}^{\prime}$ den daha düşük bir iletkenliğe sahiptir. Aromatik grafen yapısını kısmen geri yükleyerek grafen oksiti iletken hale getirmek için grafen oksidin $\mathrm{C} / \mathrm{O}$ oranının 6'nın üstüne çıkarılması gerekir.

Grafen oksit ve grafit arasında önemli farklılıklar vardır ancak her birini XRD, Raman ve FTIR ile tanımlamak mümkündür. En pratik yöntem ise XRD spektrumu ile yapılabilir; yaklaşı $2 \Theta=10$ olan XRD piki grafen okside karşılık gelirken, $2 \Theta=25-30$ civarındaki pik grafit ve grafene karşıl1k gelmektedir.

Grafen oksit, kontrol edilebilir bant aralığına, yüksek geçirgenliğe ve kontrol edilebilir iletkenliğe sahiptir ve bu nedenle GO temelli aygitların teknolojik uygulamaları ve özellikleri birçok araştırmacı tarafindan rapor edilmiştir. Örneğin, kolay ve ekonomik sprey piroliz yöntemi kullanılarak GO filminin n-InP yarı iletken üzerinde büyütülmesi ile Ti/GO/n-InP Schottky engel diyotu, Cimilli tarafından üretilmiş ve GO'in arayüzey tabakası olarak cihaz performans üzerindeki etkisi detaylı bir şekilde araştırılmıştır (Cimilli Çatır, 2020). Phan ve ark. GO ara katmanı ile $\mathrm{Al} / \mathrm{GO} / \mathrm{n}-\mathrm{Si} / \mathrm{Al}$ yapısını karanlık ve farklı $1 s ̧ ı k$ yoğunlukları altında araştırmışlardır. Akım-voltaj $(I-V)$ ölçümleri, aydınlatma yoğunluğunun artmasıly diyotun fotoakımının arttığını göstermiştir. Sonuç olarak, Al/GO/n-Si kontağının fotoiletkenlik özelliği gösterdiğini bir foto sensör olarak kullanılabileceğini belirtmişlerdir (Phan vd., 2012). Yine $\mathrm{Al} / \mathrm{GO} / \mathrm{n}-\mathrm{InP}$ ile yaptığı çalışmada Gullu ve grubu, GO arayüzey katmanı sayesinde Schottky engel yüksekliğinin geliştirilebileceğini ve bu sayede diyotun elektronik özelliklerinin büyük ölçüde değiştirilebileceğini belirtmiştir (Gullu vd., 2018).

İndiyum fosfat (InP), metal/yarı iletken (MS) Schottky kontakları, güneş pilleri ve alan etkili transistörlerin (FET'ler) üretimi için en önemli yarı iletkenlerden biridir. Ancak, düşük Schottky engel yüksekliği (SEY) (0.40-0.45 eV) gibi bir dezavantajı vardır. Bu özellik, gate elektrotu 19, 20 üzerinden büyük kaçak akıma neden olur ve doğrudan InP üzerinde FET oluşturmayı zorlaştırır. InP kontaklarının engel yüksekliğini arttırmak için, literatürde birçok araştırmacı tarafindan metal ve yarı iletken arasına çeşitli ara katmanlar yerleştirilmiş ve engelin yükseltilmesi sağlanmıştır (Çakıcı vd., 2015).

Bu çalışmada, basit ve ucuz bir yöntem olan spray pyrolysis yöntemi (Çakıcı vd., 2015) kullanılarak 
büyütülen GO filmlerinin yüzey morfolojisi ve yapısal özellikleri, soğurma ve transmittans spektrumları (UV-VIS), taramalı elektron mikroskopisi (SEM) ve X ışını kırınımı (XRD) yöntemleri ile değerlendirilmiştir. Bu çalışmanın amac1, grafen oksidin $\mathrm{Al} / \mathrm{n}$-InP diyot özellikleri üzerindeki etkisini belirlemektir. Bu nedenle, hem $\mathrm{Al} / \mathrm{GO} / \mathrm{n}$-InP MIS diyot yapısını elde etmek için hem de referans metal/n-InP kontağının engelinin arttırılması için, n-InP yarıiletken üzerinde spray pyrolysis yöntemi kullanılarak bir GO filmi büyütülmüştür. Engel yüksekliği ve idealite faktörü gibi temel diyot parametreleri elektriksel ölçümlerden elde edilerek değerlendirilmiştir ve daha sonra bu karakteristik parametreler $\mathrm{Al} / \mathrm{n}$-InP referans metal yariiletken kontak sonuçlarıyla karşılaştırılmıştır. Mevcut çalışmamızda alüminyum (Al) metali, yarı iletkene $\mathrm{n}$ tipi $\mathrm{InP}$ yariletkenine yakın bir iş fonksiyonuna sahip olduğu için ve doğru beslem sızıntı akımlarını büyük ölçüde azalttığı için Schottky kontak metali olarak seçilmiştir (Reddy vd., 2015). Ayrıca 1şık altında elektriksel ölçümler tekrarlanarak $\mathrm{Al} / \mathrm{GO} / \mathrm{n}$-InP yapının fotovoltaik özellik gösterip göstermediği araştırılmıştır.

\section{Materyal ve metot}

Al/GO/n-InP Schottky engel diyodu (SED), bu çalışmada her iki yüzü fabrikasyon olarak parlatılmış n-InP (100) yarıiletkeni üzerinde üretildi. İlk olarak, n-InP, ultrasonik banyo kullanılarak 5 dakika süreyle trikloroetilen, aseton ve metanol içinde organik kirliliklerden ve yağdan arındırıldı. Yüzeyde oluşan doğal oksit tabakasını ve olabilecek yüzeysel kirliliklerini gidermek için 1 dakika hidroflorik asit (\% 48) ve $\mathrm{H}_{2} \mathrm{O}$ çözeltisi (1:10) içinde aşındırıldı ve deiyonize su ile hızlı bir şekilde çalkalandı. Daha sonra azot gazı ile kurutulup, üzerine NANOVAK NVTH-350 yüksek vakum sisteminde yaklaşı $10^{-6}$ mbar basınçta $100 \mathrm{~nm}$ kalınlığında In ( \% 99.9) metal film oluşturuldu. Ohmik teması gerçekleştirmek için n-InP yapısı, azot gaz akışı altında 1 dakika boyunca $350^{\circ} \mathrm{C}^{\prime}$ de tavland.

Grafen oksit (GO) sentezi, modifiye edilmiş Hummers yönteminin grafitten kolay ve güvenli oksidasyon işlemi ile gerçekleştirildi (Hummers ve Offeman, 1958). $\mathrm{Bu}$ çalışmada, GO'nun hazırlanması için $120 \mathrm{~mL}$ sülfürik asit $\left(\mathrm{H}_{2} \mathrm{SO}_{4}\right)$ çözeltisine 3 g grafit tozu (\% 99 karbon, Sigma Aldrich) ilave edildi. Karışım kuvvetlice karıştırıldı ve 2 saat boyunca $100^{\circ} \mathrm{C}^{\prime}$ de tutuldu. Karışım daha sonra oda sıcaklığına soğumaya bırakıldı ve karışım bir buz banyosuna yerleştirilerek sıcaklığ $120^{\circ} \mathrm{C}$ 'nin altında tutuldu. 15 g potasyum permanganat $\left(\mathrm{KMnO}_{4}\right)$, bir saatlik bir süre zarfında yavaş yavaş ilave edildikten sonra homojen bir karışım elde etmek için bir saat daha karıştırıldı. Daha sonra buzlu su banyosundan çıkarıldı, $45^{\circ} \mathrm{C}^{\prime}$ de tutulan bir sıcak su banyosuna konuldu ve bir saat daha karıştırıldı. Bundan sonra karışım oda sıcaklığına soğumaya bırakıldı. Daha sonra karışıma $250 \mathrm{~mL}$ deiyonize su ilave edildi. Reaksiyonu durdurmak için, karışımın rengi kahverengiden siyaha yakın koyu kahverengiye dönüşünceye kadar karışıma hidrojen peroksit (yaklaşı $20 \mathrm{~mL}$ ) ilave edildi. Bu, grafen oksitin oluştuğunu gösterir. Elde edilen karışım gece boyunca dinlendirildi. Sonra, karışım santrifüjlendi ve seyreltik hidroklorik asit $(\mathrm{HCl})$ içinde süspansiyon haline getirildi. Bundan sonra $5 \mathrm{kez}$ deiyonize su ile yıkandı ve tekrar santrifüjlendi. Karışım, kalan asiti uzaklaştırmak için deiyonize suda her yıkandığında mavi turnusol kâğıdı ile asitliğinin giderildiği test edildi. Son olarak karışım süzüldü, firında kurutuldu ve toz haline getirildi.

GO filmi büyütmek için, $0.5 \mathrm{~g}$ GO tozu, GO ile yüksek dispersiyona sahip olan $20 \mathrm{ml}$ etilen glikol içine eklenerek ultrasonik olarak karıştırıldı. GO filmi Holmarc'in HD-TH-04 spray pyrolysis sistemi ile InP yariiletkenin omik kontak olmayan yüzüne büyütüldü. Başlık ve altlık arasındaki mesafe $20 \mathrm{~cm}$ ve biriktirme sicaklı̆̆ 1 bir dijital multimetre ile $200^{\circ} \mathrm{C}$ olarak belirlendi. Sıkıştırılmış ve filtrelenmiş hava taşıyıcı gaz olarak kullanıldı. Film $1 \times 1 \mathrm{~mm}^{2}$ InP yariletken altlık ve cam zerine aynı anda kapland.

GO filmin büyütülmesinden sonra, malzemeler 20 dakika boyunca $100^{\circ} \mathrm{C}$ 'de kurutuldu. Schottky diyot yapısını oluşturmak için, GO filmin üzerine $\mathrm{Al}$ (\% 99.9) metali $10^{-6}$ mbar basınç altında ve 100 $\mathrm{nm}$ kalınlığında dairesel noktalar $(0.25 \mathrm{~mm}$ yarıçap) şeklinde yüksek vakum sistemi ile buharlaştırıldı. Yüzey morfolojisi Zeiss Supra 50 VP Taramalı elektron mikroskopisi (SEM) ile karakterize edildi. Grafen oksit filmin yapisal özellikleri ile $45 \mathrm{kV}, 40 \mathrm{~mA}$ 'de CuKa $(\lambda=1.5406$ $\AA$ ) radyasyonu kullanilarak Panalitik Empyrean Xışını difraktometresi ile gerçekleştirildi. GO filminin cam altlık üzerindeki optik absorbans ve transmittans spektrumları Perkin-Elmer Lambda 2S UV-VIS spektrofotometre ile elde edildi. Al/GO/n-InP SBD'nin akım-gerilim (I-V) ölçümleri, $\pm 1 \mathrm{~V}$ aralığında Keithley 2400 akımvoltaj kaynağ 1 ve 6514 Keithley elektrometre ile alındı. Işık altındaki I-V ölçümleri için 100 $\mathrm{mW} / \mathrm{cm}^{2}$ 1şık kaynağı kullanıldı. 


\section{Bulgular}

Grafen oksit yapısı Şekil l'de görüldüğü gibi, literatürde verildiği gibi tabakalar halinde üst üste binmektedir (Olumurewa vd., 2017). Ayrica, GO filmin yarıiletken altlık yüzeyine çok iyi yapıştığı ve yariiletken yüzeyi tamamen kapladığı görülmektedir.

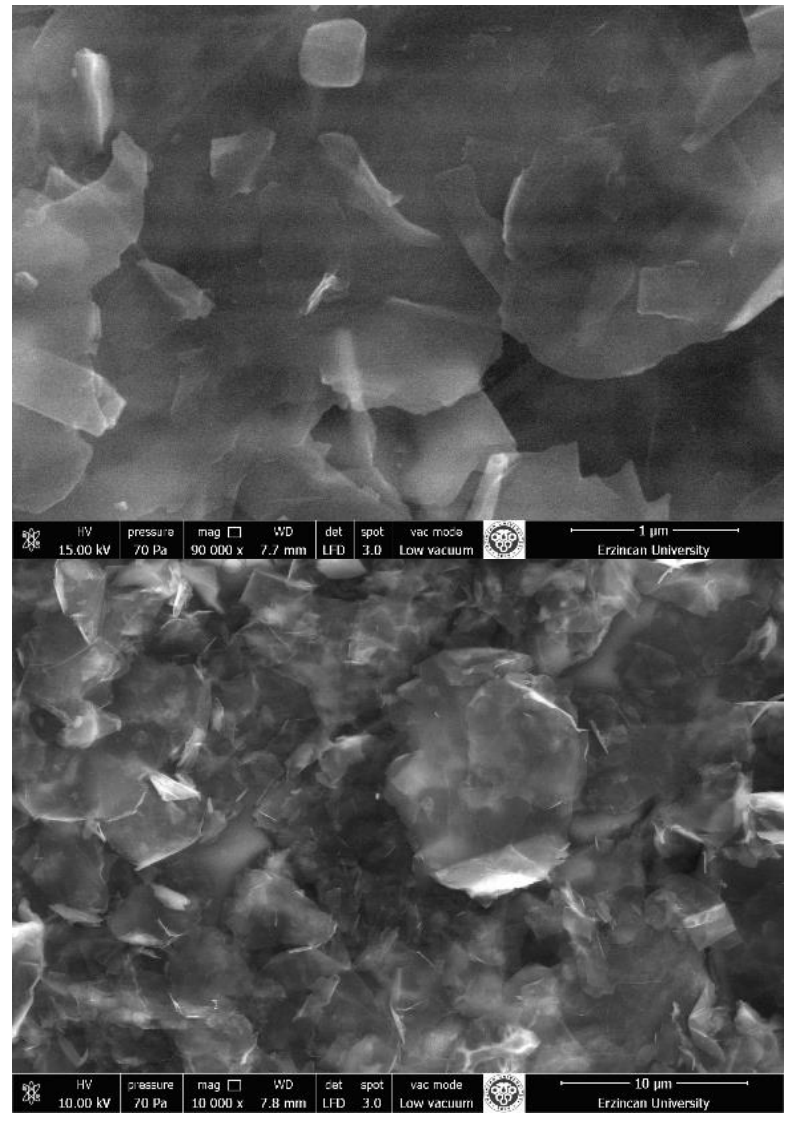

Şekil 1. GO filmin SEM görüntüleri a) $1 \mu \mathrm{m}$ b) 10 $\mathrm{nm} \mu \mathrm{m}$

Şekil 2, GO'in X-ışını kırınımı (XRD) spektrumunu göstermektedir. XRD grafiğinden grafen oksite ait karakteristik bir değer olan $2 \theta=10,5^{\circ}$ piki gözlenmiş ve $d$ mesafesi $0,83 \mathrm{~nm}$ olarak tespit edilmiştir. XRD spektrumunda görüldüğü gibi, grafen oksit filmi literatürle uygun olarak başarılı bir şekilde büyütülmüştür (Alam vd., 2017; Güllü vd., 2018). Ayrica, XRD analizinde GO malzemenin kristalin yapılasının, (002) düzleminde olduğu belirlenmiştir.

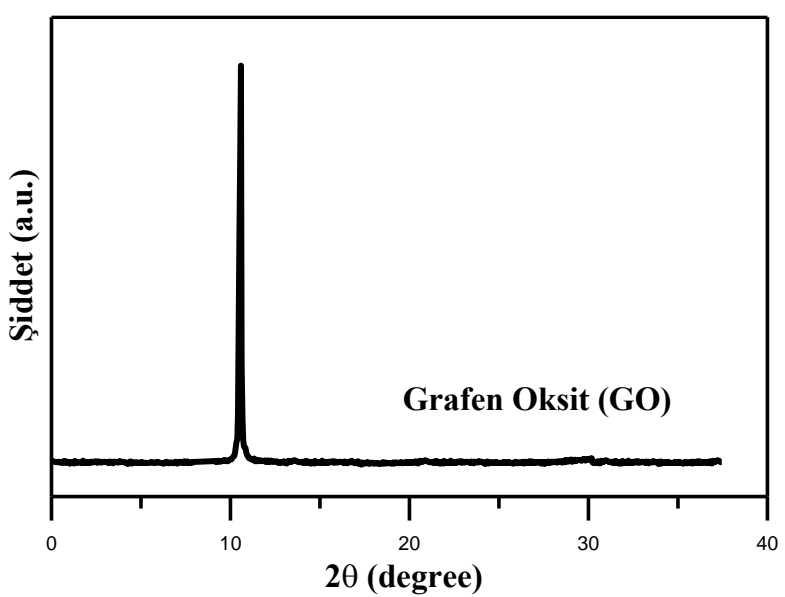

Şekil 2. GO filmin XRD spektrumu

Optik ölçümler, elektronik malzemelerin özelliklerini araştırmak için çokça tercih edilen iyi bir yöntemdir ve dalga boyunun bir fonksiyonu olarak absorbans ölçümü, malzemenin yasak enerji bant aralığ 1 hakkında önemli bilgiler verir. GO filminin absorbans ve transmittans karakteristikleri Şekil 3a-b'de verilmektedir. Absorbans spektrumu incelendiğinde, kısa dalga boylarında yüksek soğurma özelliği nedeniyle filmin opak bir malzeme olarak davrandığ söylenebilir. Filmin absorbans değerleri, literatüre uyumlu olarak yaklaşık 265 nm'de keskin bir pik yaparak 250-300 $\mathrm{nm}$ arasında genişlemiştir (Botas vd., 2013). Bu durum, foton enerjilerinin yariletken malzemenin yasak enerji bant aralığına denk geldiği veya daha büyük olduğu durumlarda valans ve iletkenlik bantları arasinda elektron transferinin gerçekleşmesiyle materyalin daha fazla soğurma yaptığını göstermektedir. Absorbans grafiği daha kısa dalga boylarında filmlerin soğurma özelliğindeki artış nedeniyle keskin bir şekilde artmıştır ve bu bölge, GO filmin temel soğurma bölgesidir. Ayrıca GO filminin optik transmittans spektrumunda görüldüğü üzere, optik geçirgenlik değerleri, artan dalga boyu ile artmış ve $700 \mathrm{~nm}$ dalga boyunda maksimum \%59.5 değerine ulaşmışır. Bununla beraber, GO filminin absorbans ve transmittans spektrumların mükemmel bir uyum içinde oldukları görülmektedir. 

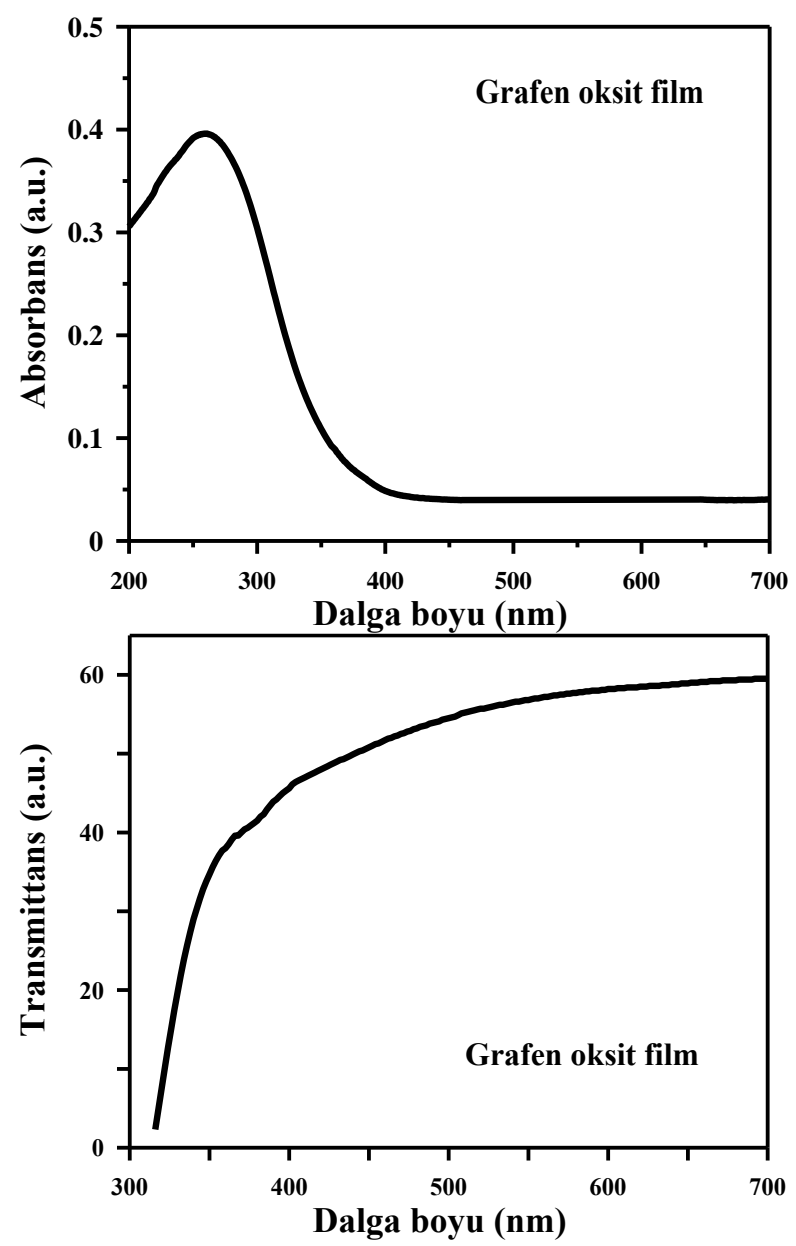

Şekil 3. GO filminin absorbans ve transmittans spektrumları

GO filmlerinin bant aralı̆̆ını belirlemek için optiksel yöntem kullanılmıştır. GO filminin soğurma karakteristiklerinden yararlanarak enerjiye $(h v)$ karşı $\left(A h v^{2}\right)$, grafiği çizilmiş ve Şekil 4'de verilmiştir. GO filminin optik bant aralığ enerjisi, $\left(A h v^{2}\right)=0$ 'daki grafiğin lineer bölgesinin enerji eksenini kestiği noktadan belirlenebilir. Absorbans spektrumlarından, GO filmin doğrudan bant boşluğuna sahip bir malzeme olduğu ve 3.36 $\mathrm{eV}$ bant aralığına sahip olduğu belirlenmiştir. Elde edilen verilere göre GO filminin güneş pili uygulamaları gibi çeşitli optoelektronik uygulamalar için uygun olduğu sonucuna varılabilir (Çatır, 2020).

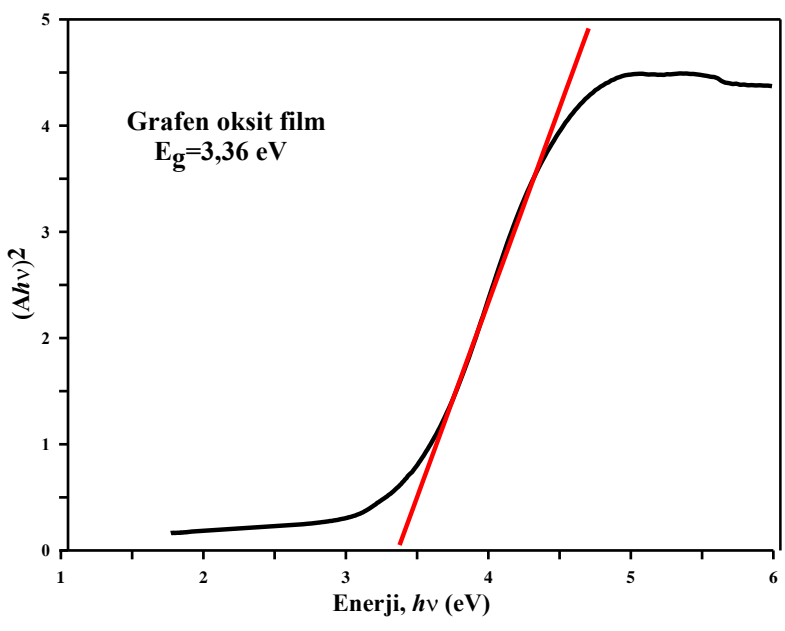

Şekil 4. GO filminin UV-VIS ölçümlerinden elde edilen $\left(A h v^{2}\right)-(h v)$ grafiği

Bir diyotun kalitesi birimi olmayan bir parametre olan idealite faktörü $(n)$ ile belirtilmektedir. İdealite faktörünün ideal bir diyot için değeri 1'e eşittir. Schottky diyotların idealite faktörü gibi temel parametrelerini hesaplamak için termiyonik I-V ölçümleri kullanılır (Cimilli vd., 2007). Bu sebeple, $\mathrm{Al} / \mathrm{GO} / \mathrm{n}-\mathrm{InP}$ diyotunun $I-V$ özelliklerini analiz etmek için termiyonik emisyon (TE) modeli kullanıldı. Bu modele göre Termiyonik akım denklemi (Rhoderick ve Williams, 1988).

$$
I=I_{0} \exp \left(\frac{q V}{n k T}\right)\left[1-\exp \left(-\frac{q V}{k T}\right)\right]
$$

burada $\mathrm{I}_{0}$ sızıntı akımıdır ve Eşitlik 2 ifadesi ile verilir.

$I_{0}=A A^{*} T^{2} \exp \left(-\frac{q \Phi_{b 0}}{k T}\right)$

$\mathrm{Bu}$ ifadede $A$ diyot alan1, $\Phi_{b 0}$ sifir beslem engel yüksekliği, $q$ elektronun yükü, $k$ Boltzmann sabiti $\left(k=8.625 \times 10^{-5} \mathrm{eV} / \mathrm{K}\right), T$ mutlak sicaklik, $A^{*}$ ise etkin Richardson sabitidir ( $n$-tipi InP için 9.4 $\mathrm{Acm}^{2} \mathrm{~K}^{2}$ ) (Cimilli vd., 2008). $\Phi_{b 0}$ aşağıdaki formülle hesaplanır:

$$
\Phi_{b 0}=\frac{k T}{q} \ln \left(\frac{A A^{*} T^{2}}{I_{0}}\right)
$$

Diyotların idealite faktörlerini $(n)$ hesaplamak için, (1) eşitliğinin her iki tarafının doğal logaritması alınır ve $V^{\gamma}$ ye göre türevi alınırsa, idealite faktörü için aşağıdaki ilişki elde edilir:

$n=\frac{q}{k T}\left(\frac{d V}{d \ln I}\right)$ 
Şekil 5, Al/GO/n-InP SE diyotunun ve $\mathrm{Al} / \mathrm{n}$-InP referans diyotunun doğru ve ters beslem yarı logaritmik I-V karakteristiklerini göstermektedir. Şekil 5'de görülebileceği gibi, $\ln I-V$ karakteristikleri GO filminin oda sıcaklığında etkisini açıkça göstermektedir ve GO arayüzey tabakası sayesinde diyotun doğrultma performans1 büyük ölçüde iyileştirilirken sızıntı akımı önemli ölçüde azalmıştır. Sızıntı akım değerindeki bu azalma, engel yüksekliğinin artmasına bağlanabilir (Baltakesmez vd., 2019). Ayrica bu durum, GO sayesinde pi bağlarının sigma bağlarına dönüşmesi suretiyle mevcut pi bağlarının azaltılmış olduğuna ve bu sayede $\mathrm{n}$-InP yarı iletken yüzeyinin pasifleştirilmesinden kaynaklandığ 1 düşünülebilir. Yine Şekil 5'den görüleceği üzere, doğru beslem $\operatorname{lnI} I V$ eğrileri geniş bir bölgede lineer olmasına rağmen gerilimin yüksek değerlerinde seri direnç ve diğer etkilerden dolayı lineerlikten sapmaktadır.

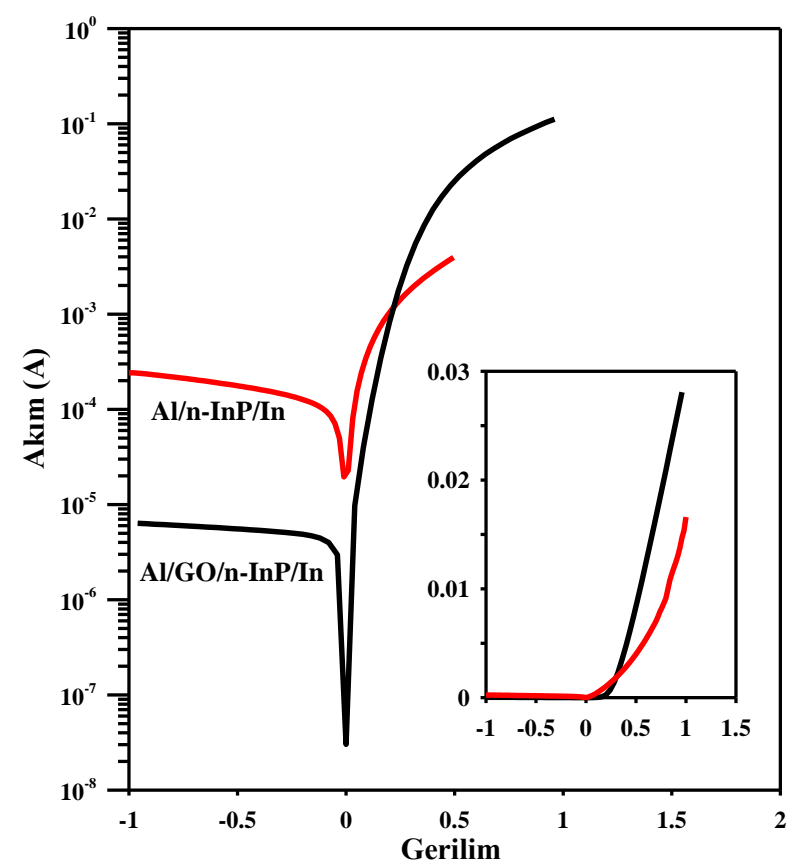

Şekil 5. Al/n-InP ve $\mathrm{Al} / \mathrm{GO} / \mathrm{n}-\mathrm{InP}$ Schottky diyotlarının oda sıcaklığında ve karanlıkta ölçülen I- $V$ karakteristikleri

$\mathrm{Al} / \mathrm{n}$-InP referans diyotu ve $\mathrm{Al} / \mathrm{GO} / \mathrm{n}-\mathrm{InP}$ diyotu için Şekil 5'den elde edilen bariyer yükseklikleri ve idealite faktörleri Tablo 1'de verilmiştir. Tablo l'de görüldüğü gibi, $\mathrm{n}$ idealite faktörü ve engel yüksekliği değerleri referans diyot için sırasıyla 1.13 ve $0.42 \mathrm{eV}$ ve GO arayüzey tabakalı diyot için ise sırasıyla 1.17 ve $0.78 \mathrm{eV}$ olarak hesaplanmıştır. Her iki diyot için de 1'den büyük bir idealite faktörü elde edildi. $\mathrm{Bu}$ sonuç engelin inhomojenliği, arayüzey durumları ve seri direncin varlığı ile açıklanabilir (Aydoğan vd., 2008). Ayrıca GO arayüzeyli numunenin referans diyota göre idealite faktörünün az da olsa arttığı ve engel yüksekliğinin ise yaklaşı iki kat artığı görülmektedir. $\mathrm{Bu}$ durum $\mathrm{GO}$ arayüzeyin varlığına atfedilmiştir. Literatürde InP Schottky kontaklarının engel yüksekliğini arttırmak için, birçok araştırmacı tarafindan metal ve yarı iletken arasına çeşitli organik veya inorganik hatta polimer yapılı ara katmanlar yerleştirilmiştir (Çakıcı vd., 2015; Çatır, 2020; Gullu vd., 2018; Reddy vd., 2016). Metal/arayüzey/yariletken diyotlar oluşturulmak suretiyle InP Schottky diyotlarının yaklaşık $0.4 \mathrm{eV}$ civarında olan engel yüksekliği değerinin $0,7-0,9$ eV'a kadar artması sağlanmıştır.
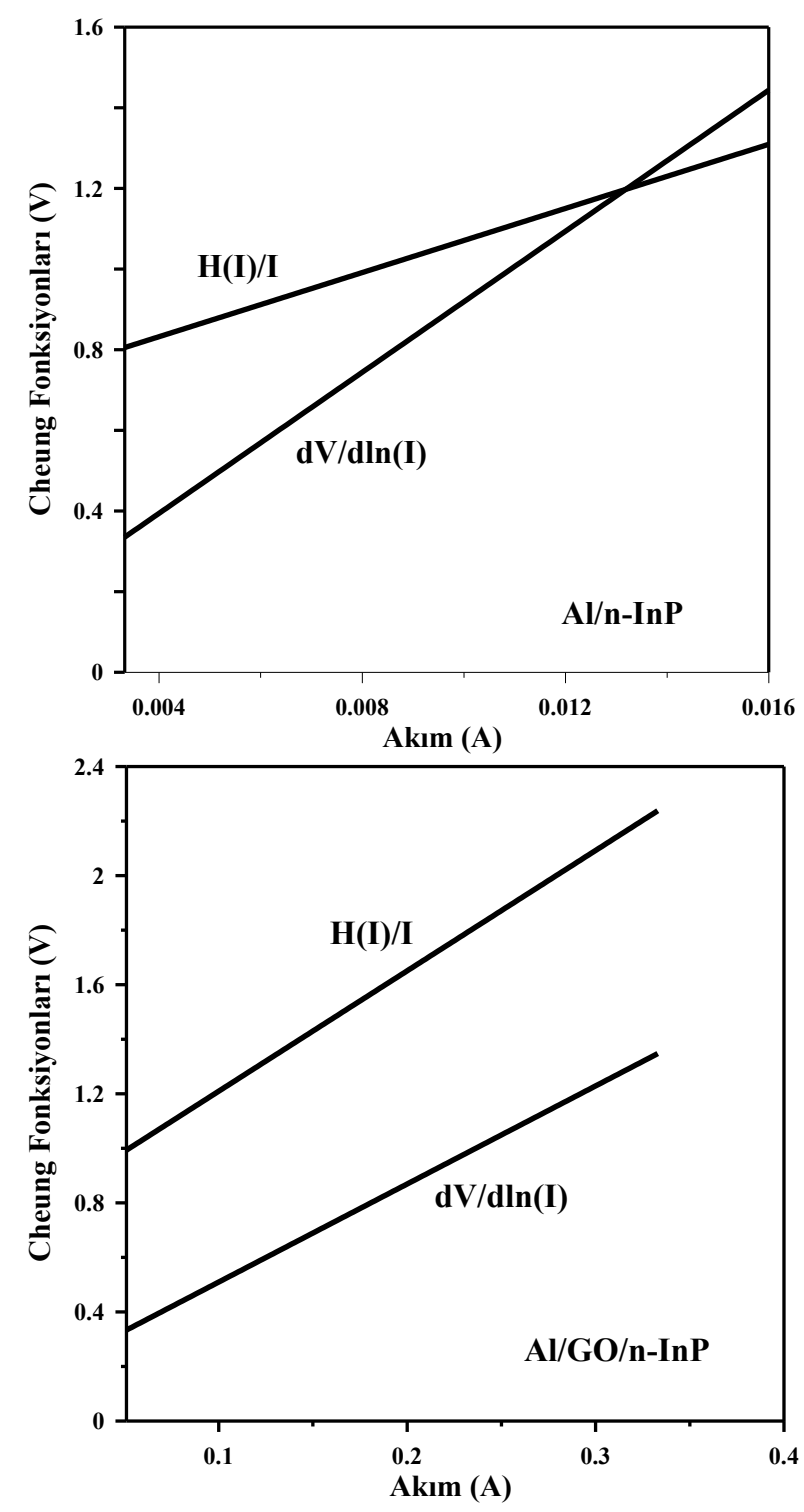

Şekil 6. $\mathrm{Al} / \mathrm{n}$-InP ve $\mathrm{Al} / \mathrm{GO} / \mathrm{n}-\mathrm{InP}$ Schottky diyotlarının Cheung fonksiyonlarından elde edilen $d V / d(\ln I)-I$ ve $H(I)-I$ grafikleri

Yukarıda bahsedildiği üzere, idealite faktörünün 1 'den daha büyük olmasının bir nedeni de, yüksek voltajlara karşılık gelen doğru beslem akım voltaj eğrilerinin lineerlikten sapmasına neden olan seri direnç $\left(R_{s}\right)$ etkisidir. Schottky diyotlarda akım- 
gerilim karakteristiğini etkileyen önemli etkenlerden biri yarıiletken gövdenin nötral bölge direncidir ve seri direnç olarak adlandırılır. Seri direnç, bir metal-yariiletken Schottky diyotunun çok önemli bir parametresidir ve yüksek gerilim değerlerinde etkilidir (Sağlam vd., 1996). Aslında Schottky diyotun direnci kontak direnci ile seri direncin kombinasyonu şeklinde düşünülebilir. Seri direnç, diyottan geçen akımı sınırlayarak akımın doyuma ulaşmasına neden olmaktadır. Böylece yüksek seri dirence sahip Schottky diyotlarda akım değeri çok küçük olmaktadır. Bu durum, üretilen metal-yariiletken Schottky diyotların özellikle yüksek akım gerektiren uygulamalarda kullanılmasına engel olmaktadır. Doğru beslem $I-V$ grafiklerinin doğrusal ve doğrusal olmayan kısımlarında iki parametre önemlidir. Bunlar $n$ ve $\Phi_{b 0}$ 'dir. $R_{s}, n$ ve $\Phi_{b 0}$, Cheung fonksiyonları yöntemi (Cheung vd., 1986) ile hesaplanmıştır (Tablo 1). Cheung fonksiyonları aşağıdaki gibi ifade edilir (Cheung vd., 1986):

$$
\frac{d V}{d(\ln I)}=\frac{n k T}{e}+I R_{s}
$$

ve

$H(I)=n \Phi_{b}+I R_{s}$

$\mathrm{Al} / \mathrm{n}$-InP referans diyotu ve $\mathrm{Al} / \mathrm{GO} / \mathrm{n}$-InP diyotu için oda sicaklığındaki $d V / d(\ln I)-I$ grafikleri ve $H(I)-I$ grafikleri, Şekil 6 ' da verilmiştir. Al/n-InP ve $\mathrm{Al} / \mathrm{GO} / \mathrm{n}-\mathrm{InP}$ diyotu için $d V / d(\ln I)-I$ grafiğinden, seri direnç $R_{s}$ değerleri sirasıyla $39.77 \Omega$ ve $4.41 \Omega$; $H(I)$-I grafiklerinden seri direnci $R_{s}$ değerleri 87.47 $\Omega$ ve7.59 $\Omega$ olarak hesaplanmıştır (Tablo1). Bu sonuçlar GO arayüzeyli diyotun seri direnç değerlerinin azaldığııı göstermektedir. Ayrıca $d V / d(\ln I)-I$ ve $H(I)-I$ fonksiyonlarından hesaplanan bu seri direnç değerlerinin birbirine yakın olduğu bulunmuştur.

Bununla birlikte, $\mathrm{Al} / \mathrm{n}-\mathrm{InP}$ ve $\mathrm{Al} / \mathrm{GO} / \mathrm{n}-\mathrm{InP}$ diyotu için $d V / d(\ln I)-I$ grafiğinden idealite faktörü değerleri sirasiyla 1.13 ve 1.59 ve $H(I)-I$ grafiklerinden engel yükseklikleri sırasıyla $0.44 \mathrm{eV}$ ve $0.77 \mathrm{eV}$ olarak hesaplanmıştır. Sonuç olarak Cheung fonksiyonları yardımıyla hesaplanan engel yüksekliği, idealite faktörü ve seri direnç değerlerinin geleneksel $I-V$ karakteristikleri ile büyük uyum içinde olduğu görülmektedir. $\mathrm{Bu}$ durum $\mathrm{Al} / \mathrm{n}$-InP ve $\mathrm{Al} / \mathrm{GO} / \mathrm{n}$-InP diyotları için yapılan hesaplamaların doğruluğunu desteklemektedir.

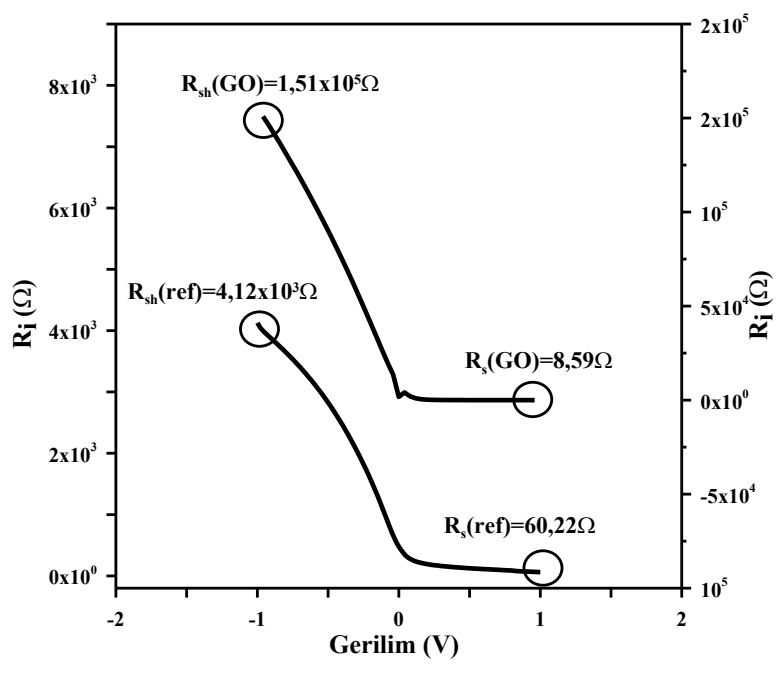

Şekil 7. $\mathrm{Al} / \mathrm{n}$-InP referans diyotu ve $\mathrm{Al} / \mathrm{GO} / \mathrm{n}-\mathrm{InP}$ diyotlarının $\mathrm{Ohm}$ kanunundan elde edilen $R_{s}$ ve $R_{s h}$ değerleri

$\mathrm{Al} / \mathrm{n}$-InP referans diyotu ve $\mathrm{Al} / \mathrm{GO} / \mathrm{n}$-InP diyotunun nötral bölgesinin direnci olan seri direnç $\left(R_{s}\right)$ ve yariiletken arayüzeyin özelliklerinden kaynaklanan şönt direnci $\left(R_{s h}\right), \quad I-V$ verileri kullanılarak Ohm Kanunu'ndan hesaplanmıştır. Şönt direnci ve seri direnç, diyotun akım-voltaj özelliklerini önemli derecede etkilemektedir. Schottky diyotlara uygulanan toplam voltaj, arayüzey tabakası, tüketim bölgesi ve dirençler arasında paylaşılır. Sonuç olarak, arayüzey tabakasının varlığına ek olarak, $R_{s}$ ve $R_{s h}$ değerleri de doğrudan cihaz kalitesini, performansını ve güvenilirliğini etkilemektedir (Çiçek vd., 2018; Çiçek vd., 2017). İdeal bir diyot için şönt direnci çok büyük olmalı ve seri direnç ise çok küçük olmalidir (Duman vd., 2015). Al/n-InP referans diyotu ve $\mathrm{Al} / \mathrm{GO} / \mathrm{n}-\mathrm{InP}$ diyotunun bağlantı direnci $R_{i}^{\prime} \operatorname{dir} \quad(R i=d V / d I) . \quad R_{s}$ ve $R_{s h}$ değerleri $R_{i}-V$ grafiklerinden oda sicaklığında $\mathrm{Al} / \mathrm{n}$-InP referans diyotu ve $\mathrm{Al} / \mathrm{GO} / \mathrm{n}$-InP diyotları için elde edilmiştir (Şekil 7). Her iki diyot için $R_{s}$ ve $R_{s h}$ arasında çok büyük bir fark olduğu görülmektedir (Tablo 1 ). $\mathrm{Al} / \mathrm{n}$-InP referans diyotu için $R_{s}=60.22 \Omega$ ve $R_{s h}=4.12 \times 10^{3} \Omega, \mathrm{Al} / \mathrm{GO} / \mathrm{n}$-InP diyotu için ise için $R_{s}=8.60 \Omega$ ve $R_{s h}=1.51 \times 10^{5} \Omega$ olarak bulunmuştur. Ayrıca referans numune ile karşılaştırıldığında $\mathrm{Al} / \mathrm{GO} / \mathrm{n}-\mathrm{InP}$ diyotunun her iki yöntemle hesaplanan seri direnç değerlerinin azaldığı, şönt direnç değerlerinin arttığı görülmektedir. $\mathrm{Bu}$ durum hem $\mathrm{GO}$ arayüzeyin $\mathrm{Al} / \mathrm{GO} / \mathrm{n}-\mathrm{InP}$ diyotunun elektriksel özelliklerine olumlu katkılarının olduğunu hem de $\mathrm{Al} / \mathrm{GO} / \mathrm{n}-\mathrm{InP}$ yapısının potansiyel elektronik uygulamalar için uygun olduğunu göstermektedir. 
Tablo 1. Al/n-InP (ref) ve Al/GO/n-InP diyotlarına ait karakteristik parametreler

\begin{tabular}{|c|c|c|c|c|c|c|c|c|}
\hline \multirow{3}{*}{ Diyot } & \multicolumn{9}{|c|}{ I- $V$ Karakteristikleri } \\
\cline { 2 - 9 } & \multicolumn{4}{|c|}{ Ohm Kanunu } & \multicolumn{4}{c|}{ Cheung-Cheung } \\
\cline { 2 - 9 } & $n$ & $\Phi_{b 0}(\mathrm{eV})$ & $R_{s}(\Omega)$ & $R_{s h}(\Omega)$ & $n$ & $R_{s}(\Omega)$ & $\Phi_{b 0}(\mathrm{eV})$ & $R_{s}(\Omega)$ \\
\hline $\mathrm{Al} / \mathrm{n}-\mathrm{InP}$ & 1.13 & 0.42 & 60.22 & $4.12 \times 10^{3}$ & 1.13 & 39.77 & 0.44 & 87.47 \\
\hline $\mathrm{Al} / \mathrm{GO} / \mathrm{n}-\mathrm{InP}$ & 1.17 & 0.78 & 8.60 & $1.51 \times 10^{5}$ & 1.59 & 4.41 & 0.77 & 7.59 \\
\hline
\end{tabular}

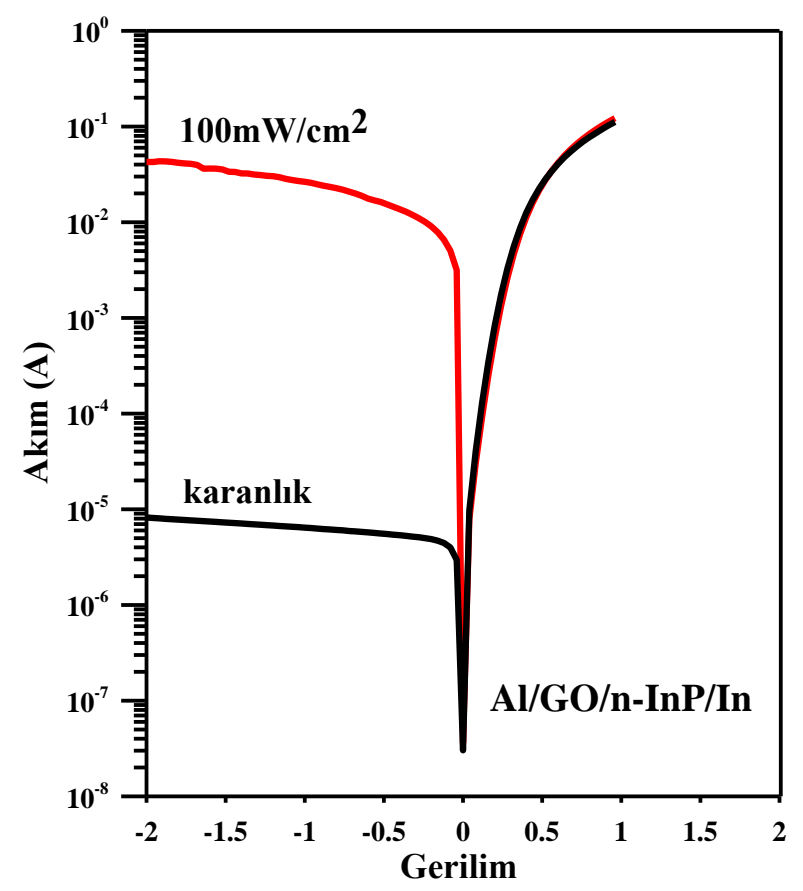

Şekil 8. Al/GO/n-InP Schottky diyotun karanlık ve $100 \mathrm{~mW} / \mathrm{cm}^{2} 1$ şı $\mathrm{k}$ ortamında $I-V$ karakteristikleri $(300 \mathrm{~K})$

Şekil 8, karanlıkta ve $100 \mathrm{~mW} / \mathrm{cm}^{2} 1$ şı ortamında elde edilen $\mathrm{Al} / \mathrm{GO} / \mathrm{n}$-InP Schottky diyotunun oda sıcaklığı $I-V$ eğrilerinin karşılaştırmasını göstermektedir. Burada, I-V grafiklerinin doğru beslem kısımları değişmeden kalmakta, ters beslem ise 1 şı etkisiyle artmaktadır. $\mathrm{Bu}$ durum, ters beslem Schottky akımının, arayüzdeki taşıyıcı yoğunluğundaki artışına atfedilen 1 şık etkisine oldukça bağlı olduğu anlamına gelir. Yani, akımdaki artış, 1şığın etkisi nedeniyle elektron ve boşluk çiftlerindeki artıştan kaynaklanır. Sonuç olarak, Al/GO/n-InP Schottky diyotun fotovoltaik özellikler gösterdiği görülmektedir ve bu durum $\mathrm{Al} / \mathrm{GO} / \mathrm{n}$-InP yapısını 1şığa duyarlı elektronik devre sistemlerinde devre elemanı olarak kullanmak için çok elverişli yapmaktadır (Çatır, 2020).

\section{Tartışma ve sonuçlar}

$\mathrm{Bu}$ çalışmada, Modifiye Hummers metodu ile üretilen grafen oksit basit ve ucuz bir yöntem olan spray pyrolysis yöntemi kullanılarak InP yarriletkeni üzerine kaplanmış ve daha sonra Al/GO/n-InP Schottky diyot yapısı elde edilmiştir. GO filminin SEM ve XRD analizleri grafen oksitin uygun şekilde kaplandığını göstermiştir. Absorbans spektrumunda 265 nm'de keskin bir pik gözlemlenmiş ve GO filmin yasak enerji aralığ 1 $3.36 \mathrm{eV}$ olarak hesaplanmıştır. Ayrıca GO filminin optik transmittans spektrumu, artan dalga boyu ile artmış ve $700 \mathrm{~nm}$ dalga boyunda maksimum $\% 59.5$ değerine ulaşmıştır. $\mathrm{Bu}$ sonuçlar $\mathrm{GO}$ filminin güneş pili uygulamaları gibi çeşitli optoelektronik uygulamalar için elverişli olduğunu göstermektedir. Deneysel $I-V$ karakteristiklerinden $\mathrm{Al} / \mathrm{GO} / \mathrm{n}$-InP diyotunun $0.78 \mathrm{eV}$ olarak hesaplanan engel yüksekliği değerinin referans numuneye göre yaklaşık iki kat arttığ görülmüştür. Cheung fonksiyonları yöntemi ile $\mathrm{Al} / \mathrm{GO} / \mathrm{n}$-InP diyotu için seri direnç $R_{s}$ değerleri sırasıyla $4.41 \Omega(d V / d(\ln I)$ $I)$ ve $7.59 \Omega(H(I)-I)$ olarak hesaplanmıştır. $R_{i}-V$ grafiklerinden oda sicaklığında $\mathrm{Al} / \mathrm{GO} / \mathrm{n}-\mathrm{InP}$ diyotu için $R_{s}$ ve $R_{s h}$ değerleri $R_{s}=8.60 \Omega$ ve $R_{s h}=1.51 \times 10^{5} \quad \Omega$ olarak bulunmuştur. Ayrica referans numune ile karşılaştırıldığında $\mathrm{Al} / \mathrm{GO} / \mathrm{n}$ InP diyotunun her iki yöntemle hesaplanan seri direnç değerlerinin azaldığ değerlerinin arttığı görülmektedir. Bu durum hem $\mathrm{GO}$ arayüzeyin $\mathrm{Al} / \mathrm{GO} / \mathrm{n}-\mathrm{InP}$ diyotunun elektriksel özelliklerine olumlu katkılarının olduğunu hem de $\mathrm{Al} / \mathrm{GO} / \mathrm{n}-\mathrm{InP}$ yapısının potansiyel elektronik uygulamalar için uygun olduğunu göstermektedir. Diğer taraftan, $100 \mathrm{~mW} / \mathrm{cm}^{2}$ 1şık ortamında elde edilen $\mathrm{Al} / \mathrm{GO} / \mathrm{n}-\mathrm{InP}$ Schottky diyotunun $I-V$ eğrilerinin ters beslem kısmı 1 ş1k etkisiyle çok büyük bir artış göstermiştir. Bunun sonucunda Al/GO/n-InP Schottky diyotun çok iyi fotovoltaik özelliklere sahip olduğu ve $\mathrm{Al} / \mathrm{GO} / \mathrm{n}$-InP yapısının 1şığa duyarlı elektronik devre sistemlerinde devre elemanı olarak kullanmak için çok elverişli olduğunu görülmektedir.

\section{Kaynaklar}

Alam, S. N., Sharma, N. and Kumar, L. (2017). Synthesis of graphene oxide (GO) by modified hummers method and its thermal reduction to obtain reduced graphene oxide (rGO), Graphene, 06(01), $1-18$, https://doi:10.4236/graphene.2017.61001 
Aydoğan, Ş., Sağlam, M. and Türüt, A. (2008). Some electrical properties of polyaniline/p-Si/Al structure at $300 \mathrm{~K}$ and $77 \mathrm{~K}$ temperatures, Microelectronic Engineering, 85(2), 278-283, https://doi:10.1016/j.mee.2007.06.004

Baltakesmez, A., Taşer, A., Kudaş, Z., Güzeldir, B., Ekinci, D. and Sağlam, M. (2019). Barrier height modification of $\mathrm{n}$-InP using a silver nanoparticles loaded graphene oxide as an interlayer in a wide temperature range. Journal of Electronic Materials, $\quad 48(5), \quad 3169-3182$, https://doi:10.1007/s11664-019-07088-8

Botas, C., Álvarez, P., Blanco, P., Granda, M., Blanco, C., Santamaría, R. and Menéndez, R. (2013). Graphene materials with different structures prepared from the same graphite by the Hummers and Brodie methods. Carbon, 65, 156-164, https://doi:10.1016/j.carbon.2013.08.009

Chen, C., Xu, K., Ji, X., Miao, L. and Jiang, J. (2014). Enhanced adsorption of acidic gases $\left(\mathrm{CO}_{2}, \mathrm{NO}_{2}\right.$ and $\mathrm{SO}_{2}$ ) on light metal decorated graphene oxide. Physical Chemistry Chemical Physics, 16(22), 11031-11036.

Cheung, S. K., Cheung, N. W. and H., N. (1986). Extraction of Schottky diode parameters from forward current-voltage characteristics. Applied Physics Letters, 49(2), 85-87, https://doi:10.1063/1.97359.

Cimilli Çatır, F. E. (2020). Fabrication and characterization of Au/n-type InP Schottky barrier diode with monolayer graphene interlayer. Semiconductor Science and Technology, 35(3), 035023. https://doi:10.1088/1361-6641/ab6bb4

Cimilli Çatır, F. E. (2020). The structural, optical, and electrical characterization of Ti/n-InP Schottky diodes with graphene oxide interlayer deposited by spray pyrolysis method. Physica Status Solidi (a), 217(19), 2000125. https://doi:https://doi.org/10.1002/pssa.2020001 25

Cimilli, F. E., Efeoğlu, H., Sağlam, M. and Türüt, A. (2008). Temperature-dependent current-voltage and capacitance-voltage characteristics of the Ag/n-InP/In Schottky diodes. Journal of Materials Science: Materials in Electronics, 20(2), 105-112. https://doi:10.1007/s10854-0089635-Z

Cimilli, F. E., Sağlam, M. and Türüt, A. (2007). Determination of the lateral barrier height of inhomogeneous Au/n-type InP/In Schottky barrier diodes. Semiconductor Science and Technology, 22(8), 851-854. https://doi:10.1088/0268-1242/22/8/003
Çakıcı, T., Sağlam, M. and Güzeldir, B. (2015). The comparison of electrical characteristics of $\mathrm{Au} / \mathrm{n}$ $\mathrm{InP} / \mathrm{In}$ and $\mathrm{Au} / \mathrm{In} 2 \mathrm{~S} 3 / \mathrm{n}$-InP/In junctions at room temperature. Materials Science and Engineering: $\quad B, \quad 193, \quad 61-69$. https://doi:10.1016/j.mseb.2014.11.003

Çiçek, O., Tan, S. O., Tecimer, H. and Altındal, Ş. (2018). Role of graphene-doped organic/polymer nanocomposites on the electronic properties of Schottky junction structures for photocell applications. Journal of Electronic Materials, 47(12), 7134-7142. https://doi:10.1007/s11664018-6644-4.

Çiçek, O., Uslu Tecimer, H., Tan, S. O., Tecimer, H., Orak, İ. and Altındal, Ş. (2017). Synthesis and characterization of pure and graphene (Gr)doped organic/polymer nanocomposites to investigate the electrical and photoconductivity properties of $\mathrm{Au} / \mathrm{n}-\mathrm{GaAs}$ structures. Composites Part B: Engineering, 113, 14-23. https://doi:10.1016/j.compositesb.2017.01.012.

Dimiev, A. M. and Eigler, S. (2016). Graphene oxide: fundamentals and applications. United Kingdom: John Wiley \& Sons.

Dimiev, A. M. and Tour, J. M. (2014). Mechanism of graphene oxide formation, ACS Nano, 8(3), 3060-3068

Duman, S., Ozcelik, F. S., Gürbulak, B., Gülnahar, M. and Turut, A. (2015). Current-voltage and capacitance-conductance-voltage characteristics of $\mathrm{Al} / \mathrm{SiO}_{2} / \mathrm{p}-\mathrm{Si}$ and $\mathrm{Al} /$ methyl green $(\mathrm{MG}) / \mathrm{p}-\mathrm{Si}$ structures. Metallurgical and Materials Transactions A, 46(1), 347-353. https://doi:10.1007/s11661-014-2621-6

Gullu, O., Cankaya, M. and Rajagopal Reddy, V. (2018). Barrier enhancement of $\mathrm{Al} / \mathrm{n}$-InP Schottky diodes by graphene oxide thin layer. Indian Journal of Physics, 93(4), 467-474, https://doi:10.1007/s12648-018-1311-4

Hummers, W. S. and Offeman, R. E. (1958). Preparation of graphitic oxide. Journal of the American Chemical Society, 80(6), 1339-1339, https://doi:10.1021/ja01539a017

Li, F., Jiang, X., Zhao, J. and Zhang, S. (2015). Graphene oxide: A promising nanomaterial for energy and environmental applications. Nano Energy, 16, 488-515.

Olumurewa, K. O., Olofinjana, B., Fasakin, O., Eleruja, M. A. and Ajayi, E. O. B. (2017). Characterization of high yield graphene oxide synthesized by simplified Hummers method. Graphene, 06(04), 85-98. https://doi:10.4236/graphene.2017.64007 
Park, S. and Ruoff, R. S. (2009). Chemical methods for the production of graphenes. Nature nanotechnology, 4(4), 217.

Perrozzi, F., Prezioso, S. and Ottaviano, L. (2014). Graphene oxide: from fundamentals to applications. Journal of Physics: Condensed Matter, 27(1), 013002, https://doi:10.1088/0953$8984 / 27 / 1 / 013002$

Phan, D. T., Gupta, R. K., Chung, G. S., Al-Ghamdi, A. A., Al-Hartomy, O. A., El-Tantawy, F. and Yakuphanoglu, F. (2012). Photodiodes based on graphene oxide-silicon junctions. Solar Energy, 86(10), 2961-2966, https://doi:https://doi.org/10.1016/j.solener.2012 .07 .002

Reddy, P. R. S., Janardhanam, V., Jyothi, I., Yuk, S. H., Reddy, V. R., Jeong, J. C. and Choi, C. J. (2016). Modification of Schottky barrier properties of Ti/p-type InP Schottky diode by polyaniline (PANI) organic interlayer. JSTS: Journal of Semiconductor Technology and Science, 16(5), 664-674. https://doi:10.5573/jsts.2016.16.5.664

Reddy, V. R., Reddy, Y. M., Padmasuvarna, R. and Narasappa, T. L. (2015). Ru/Ti Schottky contacts on n-type In-P (100): temperature dependence of current-voltage (I-V) characteristics. Procedia Materials Science, 10, 666-672. https://doi:10.1016/j.mspro.2015.06.060

Rhoderick, E. H. and Williams, R. H. (1988). Metalsemiconductor contact. New York: Clarendon Press; Oxford University Press.

Sağlam, M., Ayyıldız, E., Gümüş, A., Türüt, A., Efeoğlu, H. and Tüzemen, S. (1996). Series resistance calculation for the Metal-InsulatorSemiconductor Schottky barrier diodes. Applied Physics A: Materials Science \& Processing, 62(3), https://doi:10.1007/s003390050297

Sattler, K. D. (2016). Carbon Nanomaterials Sourcebook: Graphene, Fullerenes, Nanotubes, and Nanodiamonds. New York: CRC Press.

Zhao, J., Liu, L. and Li, F. (2015). Graphene oxide: physics and applications. Verlag Berlin Heidelberg: Springer. 